\title{
Energy positivity, non-renormalization, and holomorphy in Lorentz-violating supersymmetric theories
}

\author{
Adam B. Clark \\ Department of Physics, Muhlenberg College, \\ 2400 Chew St., Allentown, PA 18104, U.S.A. \\ E-mail: aclark@muhlenberg.edu
}

ABSTRACT: This paper shows that the positive-energy and non-renormalization theorems of traditional supersymmetry survive the addition of Lorentz violating interactions. The Lorentz-violating coupling constants in theories using the construction of Berger and Kostelecky must obey certain constraints in order to preserve the positive energy theorem. Seiberg's holomorphic arguments are used to prove that the superpotential remains non-renormalized (perturbatively) in the presence of Lorentz-violating interactions of the Berger-Kostelecky type. We briefly comment on Lorentz-violating theories of the type constructed by Nibbelink and Pospelov to note that holomorphy arguments offer elegant proofs of many non-renormalization results, some known by other arguments, some new.

KeYwords: Space-Time Symmetries, Supersymmetric Effective Theories

ArXiv EPRINT: 1303.0335 


\section{Contents}

1 Introduction $\quad 1$

2 Non-renormalization of Berger-Kostelecký models by holomorphy 2

2.1 Review of Seiberg's proof by holomorphy in standard supersymmetric theories 2

2.2 Berger-Kostelecky Lorentz violation 3

2.3 Non-renormalization in Berger-Kostelecky theories 6

$\begin{array}{lll}2.4 & \text { Robustness against coordinate transformations } & 6\end{array}$

2.4.1 Extended supersymmetry and Berger-Kostelecký Lorentz violation 8

2.4.2 A manifestly non-trivial theory with BK-type Lorentz violation 9

3 Energy positivity in the BK construction $\quad 10$

$\begin{array}{lll}3.1 \text { Constraint from spin } 1 / 2 \text { particles at rest } & 10\end{array}$

$\begin{array}{lll}3.2 & \text { Constraints from scalar particles } & 12\end{array}$

$\begin{array}{lll}3.3 & \text { Constraints from moving particles } & 12\end{array}$

$\begin{array}{lll}\text { 3.3.1 General form of constraint } & 12\end{array}$

$\begin{array}{lll}3.3 .2 & \text { Boosted particles } & 13\end{array}$

$\begin{array}{lll}\text { 3.3.3 Enforcing the mass shell or dispersion relation } & 14\end{array}$

$\begin{array}{lll}3.4 & \text { An alternate view on the positive energy constraints } & 15\end{array}$

4 Non-renormalization of Nibbelink-Pospelov type LV theories 15

$\begin{array}{lll}4.1 \text { Review of Nibbelink-Pospelov construction } & 15\end{array}$

$\begin{array}{lll}4.2 & \text { Non-renormalization in NP-type theories } & 16\end{array}$

$\begin{array}{lll}4.3 & \text { Berger-Kostelecký models with charged matter } & 18\end{array}$

4.4 A comment on the possibility of SUSY-scale suppression of LV couplings 18

$\begin{array}{llr}5 & \text { Conclusion } & 19\end{array}$

\section{Introduction}

By employing the holomorphic arguments of Intriligator, Leigh, and Seiberg [3], one can show that the full non-renormalization theorems of $\mathcal{N}=1$ supersymmetry apply unaltered to theories with Lorentz violating (LV) interactions of either the Berger-Kostelecky (BK) type [1] or the Nibbelink-Pospelov (NP) type [2]. The essential point of the proof is that Lorentz symmetry plays no direct role in the holomorphy argument. As long as the normal rules of $\mathcal{N}=1$ SUSY are followed when constructing the model, and as long as the LV interaction creates no new anomalies or other surprises, then the superpotential will be protected against perturbative quantum corrections, and under appropriate conditions an exact expression for the quantum effective superpotential can be obtained, using nowstandard arguments from [3]. 
The rest of the paper is organized as follows: first, we review the general holomorphy arguments for non-renormalization in supersymmetric theories. Next we examine BK-type theories, demonstrating that they satisfy the conditions of Seiberg's holomorphy argument. Third, we show that BK-type theories require additional constraints on the values of the LV coupling constant in order for the positive energy theorem to hold. Next, we comment briefly on NP-type theories, explaining how holomorphy arguments more or less automatically prove that superpotential LV couplings and potentially divergent FI terms are protected against perturbative corrections. Holomorphy arguments go one step farther, and prove that the NSVZ $\beta$-function (in holomorphic coupling) remains subject only to one-loop renormalization and that NP-type LV couplings that enter into the gauge-kinetic function are immune to perturbative renormalization (but still subject to wavefunction renormalization). Finally, we summarize and conclude.

\section{Non-renormalization of Berger-Kostelecký models by holomorphy}

\subsection{Review of Seiberg's proof by holomorphy in standard supersymmetric theories}

The arguments of Seiberg et al. [3] hinge on three key points: 1) respect of symmetries, 2) holomorphy of the superpotential, and 3) the fact that holomorphic functions are completely determined by their singularities and asymptotic behavior [9]. All tree-level couplings in the superpotential are treated as auxiliary fields, or fully-fledged chiral superfields that just happen to be non-dynamical. A coupling that explicitly breaks a global symmetry of the rest of the theory in turn provides a selection rule constraining quantum corrections: since symmetry-breaking terms in the quantum effective potential must ultimately descend from tree-level breaking terms, we can employ the usual "that which is not forbidden is compulsory" algorithm simply by pretending that the coupling itself transforms in just the right way to preserve the broken symmetry. This provides a simple check on whether symmetry-breaking terms in the effective superpotential are consistent with the tree-level breaking terms. This is how Seiberg's prescription respects all symmetries, even the broken ones $[3,9]$. Lorentz-violating theories themselves almost invariably employ that technique for the LV couplings $[1,5]$. In much of the Lorentz-violating literature, these transformation properties of the LV couplings are dubbed "observer Lorentz invariance." See $[4,5]$ for detailed discussions. In the recent work of [27], native to the AdS/CFT correspondence, this phenomenon is referred to more simply as diffeomorphism invariance.

Holomorphy of the superpotential is a proxy condition for invariance under supersymmetry, given that one is constructing a theory using the formalism of superfields. In some sense, this is just another symmetry to respect, but this symmetry is powerful enough to deserve special mention. Supersymmetry is so restrictive that it enables divergence cancellations in 1-loop diagrams in the traditional, pre-Seiberg proofs of non-renormalization. Part of Seiberg's great insight was that holomorphicity could be taken literally and was every bit as restrictive mathematically as supersymmetry invariance is physically. This leads to point 3 , which is the punchline: respect of symmetries makes it possible to write 
down the most general holomorphic function of couplings and superfields for the superpotential. Many coefficients are fixed outright by the requirement of holomorphy. Still more coefficients can be obtained by analyzing the theory in some appropriate limit, since holomorphic functions are completely determined by their singularities and their asymptotics. Often these constraints will completely determine the superpotential $[3,9]$.

\subsection{Berger-Kostelecky Lorentz violation}

Even spacetime symmetries could be viewed by a model-builder as "just another set of symmetries." In the BK-type theories, spacetime symmetries are altered by broken Lorentz invariance, and the superalgebra is modified $[1,6]$. In NP-type theories, spacetime symmetries are altered, but the superalgebra is not $[2,7]$. In both cases, the theory can still be described in terms of superfields, and the superpotential is a holomorphic function of said superfields. In the BK-construction these superfields are not necessarily the same as the chiral and vector superfields used in traditional SUSY. Whether or not the superalgebra is modified, invariance under the (possibly modified) supersymmetry is still encoded in the holomorphy of the superpotential.

Berger and Kostelecky begin with an ordinary Wess-Zumino model, then add Lorentzviolating interactions to the Kähler potential. ${ }^{1}$ They then show that the resulting Lagrangian is almost invariant under ordinary supersymmetry but becomes completely invariant (up to total derivative terms) under slightly modified SUSY transformations [1]. Fermion and boson propagators are modified in the Lorentz-violating theories, but they retain the parallel structure which is essential for brute-force proofs of divergence cancellation in traditional SUSY theories, leading [1] to very plausibly assert that those divergences should still cancel. Berger and Kostelecky construct modified chiral superfields for their LV SUSY theories, which we will exploit to concisely prove that Berger and Kostelecky were correct about the non-renormalization theorem and divergence cancellation.

Berger and Kostelecky construct LV theories using Majorana spinors following the notation conventions of Wess and Bagger's seminal work [14]. We begin by first rewriting in the slightly more modern notation of [15] and writing an LV Wess-Zumino model for a chiral multiplet with Weyl spinors rather than Majorana. Our chiral superfield for normal SUSY theories is

$$
\begin{aligned}
\Phi= & \phi(x)+i \theta^{\dagger} \bar{\sigma}^{\mu} \theta \partial_{\mu} \phi(x)+\frac{1}{4} \theta \theta \theta^{\dagger} \theta^{\dagger} \partial_{\mu} \partial^{\mu} \phi(x) \\
& +\sqrt{2} \theta \psi(x)-\frac{i}{\sqrt{2}} \theta \theta \theta^{\dagger} \bar{\sigma}^{\mu} \partial_{\mu} \psi(x)+\theta \theta F(x) .
\end{aligned}
$$

The usual Wess-Zumino Langragian in superfield form is given by

$$
\mathcal{L}_{W Z}=\int d^{4} \theta \Phi^{*} \Phi+\int d^{2} \theta W(\Phi)+c . c .
$$

\footnotetext{
${ }^{1}$ While [1] does not use this term explicitly, they do point out that their LV interactions do not affect the superpotential. This is not obvious, since their construction involves modifying the superfields rather than adding an LV interaction constructed out of superfields. We clarify this point in section 2.2.
} 
with

$$
W(\Phi)=\frac{M}{2} \Phi \Phi+\frac{g}{3} \Phi \Phi \Phi .
$$

More general theories could be constructed by promoting $W$ to an arbitrary holomorphic function of $\Phi$ and replacing $\Phi^{*} \Phi$ with a more general Kähler potential. To facilitate contact with the work of Berger and Kostelecky, we expand the basic Wess-Zumino lagrangian as

$$
\begin{aligned}
\mathcal{L}_{W Z}= & -\partial^{\mu} \phi^{*} \partial_{\mu} \phi+i \psi^{\dagger} \bar{\sigma}^{\mu} \partial_{\mu} \psi+F^{*} F \\
& +\left(-\frac{1}{2} M \psi^{2}+M \phi F-\frac{1}{2} g \phi \psi \psi\right)+c . c .
\end{aligned}
$$

In the conventional picture (i.e. without using superfields), Lorentz-Violating interactions are added in the form of the following term, $\mathcal{L}_{L V}[1]$

$$
\begin{aligned}
\mathcal{L}_{L V}= & 2 k_{\mu \nu} \partial^{\mu} \phi^{*} \partial_{\nu} \phi+k_{\mu \nu} k_{\rho}^{\mu}\left(\partial^{\nu} \phi^{*} \partial^{\rho} \phi\right) \\
& +\frac{i}{2} k_{\mu \nu} \psi^{\dagger} \bar{\sigma}^{\mu} \partial^{\nu} \psi
\end{aligned}
$$

which can also be obtained from the original Lagrangian by replacing the derivative operator with a so-called "twisted" derivative operator [6]:

$$
\tilde{\partial}_{\mu}=\left(\delta_{\mu}^{\alpha}+k_{\mu}^{\alpha}\right) \partial_{\alpha}
$$

This operator is also denoted by $\nabla_{m}$ in [18]. Indeed, many quantities in conventional theories can be extended to BK theories by the replacement $\partial_{\mu} \rightarrow \tilde{\partial}_{\mu}$ and "twisting" all vector indices by the $\delta_{\mu}^{\alpha}+k_{\mu}^{\alpha}$ operator used in (2.6) [6]. This "folk theorem" extends to superfields, as we see when looking at the LV version of the chiral superfield [1]:

$$
\begin{aligned}
\Phi= & \phi(x)+i \theta^{\dagger} \bar{\sigma}^{\mu} \theta\left(\partial_{\mu}+k_{\mu \alpha} \partial^{\alpha}\right) \phi(x)+\sqrt{2} \theta \psi(x) \\
& +\frac{1}{4} \theta \theta \theta^{\dagger} \theta^{\dagger}\left(\partial_{\mu}+k_{\mu \alpha} \partial^{\alpha}\right)\left(\partial^{\mu}+k^{\mu \beta} \partial_{\beta}\right) \phi(x) \\
& -\frac{i}{\sqrt{2}} \theta \theta \theta^{\dagger} \bar{\sigma}^{\mu}\left(\partial_{\mu}+k_{\mu \alpha} \partial^{\alpha}\right) \psi(x)+\theta \theta F(x) .
\end{aligned}
$$

Building the LV interaction terms into a change of the superfield itself obfuscates the nature of the LV interaction as belonging to the superpotential or the Kähler potential. In [1] it is noted in passing that the LV interaction does not affect the superpotential. To understand this, note that the LV coupling $k_{\mu \nu}$ appears only in terms including both $\theta$ and $\theta^{\dagger}$; therefore, since the superpotential will only be integrated $\int d^{2} \theta$ or $\int d^{2} \theta^{\dagger}, k_{\mu \nu}$ will never appear in the action in a term born of the superpotential. Thus, the LV interactions are best thought of as part of the Kähler potential in the BK-construction.

When the full Lagrangian for the Lorentz-violating Wess-Zumino model with one chiral multiplet is written by adding up the various pieces of the Lagrangian (equations (2.5) and (2.4)) in conventional notation or by using the normal superfield Lagrangian (2.2) but with the modified LV superfields, the resulting theory is not quite invariant under normal SUSY transformations [1]. If one modifies the superalgebra and SUSY transformations by the same prescription of "twisting" the derivative operator, then the modified Lagrangian 
is invariant (up to total derivative) under the modified SUSY transformations [1]. In summary, the Lagrangian $\mathcal{L}=\mathcal{L}_{W Z}+\mathcal{L}_{L V}$ is invariant under SUSY generators $Q$ and $Q^{\dagger}$ with superspace representations

$$
\begin{aligned}
Q & =i \partial_{\theta}-\sigma^{\mu} \theta^{\dagger} \partial_{\mu}-k_{\mu \nu} \sigma^{\mu} \theta^{\dagger} \partial^{\nu} \\
Q^{\dagger} & =i \partial_{\theta^{\dagger}}-\bar{\sigma}^{\mu} \theta \partial_{\mu}-k_{\mu \nu} \bar{\sigma}^{\mu} \theta \partial^{\nu}
\end{aligned}
$$

and anti-commutation relation:

$$
\left\{Q, Q^{\dagger}\right\}=2 \sigma^{\mu} \partial_{\mu}+2 k_{\mu \nu} \sigma^{\mu} \partial^{\nu}
$$

where $\sigma^{0}$ and $\bar{\sigma}^{0}$ are each the $2 \times 2$ identity matrix, $\sigma^{i}$ is the $i$ th Pauli spin matrix, and $\bar{\sigma}^{i}=-\sigma^{i}$. We will strive to avoid the need for tracking spinor indices as much as possible, but when unavoidable we follow [15]. In brief, undotted Greek indices from the beginning of the alphabet $(\alpha, \beta, \ldots)$ denote left-handed Weyl spinor indices while their dotted counterparts denote right-handed Weyl spinor indices $(\dot{\alpha}, \dot{\beta}, \ldots)$. Spinor indices are implicitly raised and lowered as needed with the two-index Levi-Civita $\varepsilon$. Our only exception to leaving spinor indices implicit is the gauge superfield strength, $W_{\alpha}$, which we write out to distinguish from the superpotential, $W$.

There are some trivial but potentially confusing differences in notation. Berger and Kostelecky use $\theta$ and $\bar{\theta}$ where we use $\theta^{\dagger}$ and $\theta$, respectively. Invariance under the modified SUSY transformations proceeds the same with Majorana or with Weyl spinors, so we do not repeat the proof of invariance from [1]. Similar constructions exist for supersymmetric gauge theories, and we will quote results from these theories only as needed. The main difference between the spinor conventions of [15] and [1] is that the former removes the need for awkward-looking left- and right-handed projection operators involving $\gamma^{5}$ by working with Weyl-spinors so that undaggered spinors are implicitly left-handed and daggered spinors right-handed.

The BK-construction for SUSY gauge theories is constructed similarly [6]. When writing out the vector superfield in terms of component fields, simply "twist" each spacetime index on a field or derivative operator with the $\left(\delta_{\mu}^{\alpha}+k_{\mu}^{\alpha}\right)$ operator. Recasting the results of [6] with Weyl-spinors instead of Dirac we get

$$
\begin{aligned}
V & =\theta^{\dagger} \bar{\sigma}^{\mu} \theta\left(\delta_{\mu}^{\nu}+k_{\mu}{ }^{\nu}\right) A_{\nu}+\theta^{\dagger} \theta^{\dagger} \theta \lambda+\frac{1}{2} \theta \theta \theta^{\dagger} \theta^{\dagger} D \\
W_{\alpha} & =-\frac{1}{4} D^{\dagger} D^{\dagger} D_{\alpha} V,
\end{aligned}
$$

where the supercovariant derivatives are also twisted by the $\delta+k$ operator: $D_{\alpha}=\partial_{\theta^{\alpha}}-$ $i\left(\sigma^{\mu} \theta^{\dagger}\right)_{\alpha}\left(\delta_{\mu}{ }^{\nu}+k_{\mu}{ }^{\nu}\right) \partial_{\nu}$. The pure gauge Lagrangian is then the usual superspace integral of $W_{\alpha} W^{\alpha}$. This can be generalized to the non-Abelian case in the usual way. We emphasize that in this construction, the LV interactions live entirely in the gauge-kinetic function, in contrast to the original BK-model with only chiral multiplets, where the LV interaction was implicitly part of the Kähler potential. 


\subsection{Non-renormalization in Berger-Kostelecky theories}

As discussed above, supersymmetric BK theories can be constructed out of modified superfields with a superpotential which is an arbitrary holomorphic function of those modified superfields [1], as with ordinary SUSY. Holomorphy of the superpotential now encodes invariance under the modified superalgebra. Seiberg's holomorphy arguments $[3,9]$ then apply in full, as they don't reference a specific form of SUSY but more generally whatever (super)symmetry is "proxied" by holomorphy. Non-supersymmetric Lorentz-violating theories have been shown to be renormalizable in cases of pure gauge [12], in QCD [11], and in the electroweak sector [10]. Additionally, the renormalization of LV $\phi^{4}$ theory has been worked out to all orders, and renormalization of LV Yukawa theories has been solved to one-loop order [17]. We conclude from this litany of examples that nothing intrinsic to LV interactions impedes the standard program of renormalization. Furthermore, BK-type LV interactions are not chiral in nature and do not introduce any additional fermions, so they are not expected to produce new anomalies. We therefore conclude that the results of [3] apply to supersymmetric BK theories. It is worth noting that a brute force calculation using supergraphs has been carried out in [13] for BK theories with diagonal $k_{\mu \nu}$, confirming the original suspicions of [1] and proving non-renormalization in the special case of diagonal $k_{\mu \nu}$.

Our holomorphy argument goes further and shows that all the non/renormalization results of traditional SUSY apply to all supersymmetric BK theories: the superpotential is not renormalized at any order in perturbation theory, although it may be subject to renormalization through instantons or other non-perturbative effects. Additionally, such non-perturbative renormalization can often be computed using the methods of [3]. With Wess-Zumino models, such as studied in [1], it is quite well known that Seiberg's arguments prove the tree-level superpotential is exact. We have shown that this continues in the presence of LV interactions, and this proof opens the door to further Seiberg-style analysis of BK-type LV extensions to the MSSM.

The non-renormalization theorem goes beyond the LV Wess-Zumino model. Vector superfields for BK-type theories were constructed in [6]. As with chiral superfields in [1], the prescription was to "twist" the derivative operator and all space-time indices. Also as with chiral superfields, the LV coupling appears only in terms with both $\theta$ and $\theta^{\dagger}$, so the LV interaction is most properly thought of as part of the Kähler potential. The holomorphy argument is identical to the chiral superfield case. Furthermore, since practically any $\mathcal{N}=1$ SUSY theory can be built with a collection of vector and chiral superfields with various interactions, our proof of non-renormalization for BK-type theories extends quite broadly. It is important to note, however, that the LV coupling, as part of the Kähler potential in BK-type theories, is not protected against renormalization.

\subsection{Robustness against coordinate transformations}

A cautionary note has been pointed out numerous times $[2,6]$ that the BK-type LV interactions can be absorbed into the metric by the coordinate transformation $x^{\mu \prime}=x^{\mu}-k_{\nu}^{\mu} x^{\nu}$. It is argued in [6] that this coordinate transformation causes Lorentz-violation to manifest 
itself in peculiarities of the coordinate system, namely non-orthogonality. Nevertheless, BK-type LV interactions could be realized outside the metric in a different setting. Any theory with extended SUSY and multiple sectors, one with BK-type LV and one respecting Lorentz symmetry, would be immune to complete removal of the LV interaction. In such a setup, the coordinate transformation to undo the LV interaction in one sector would reintroduce it in the other sector. A simple demonstration is $\mathcal{N}=2$ gauge theory with one hypermultiplet where BK-type LV interactions exist for only one of the two $\mathcal{N}=1$ chiral multiplets that comprise hypermultiplet. The BK-type LV interaction would partially break the SUSY down to $\mathcal{N}=1$, but attempting to undo the LV interaction with a coordinate transformation would then swap the roles of the two multiplets. Similar constructions have been outlined for $\mathcal{N}=1$ supersymmetry in [6] and in [13] where the two sectors interact only via soft SUSY-breaking terms. Analogous constructions could be used to partially break $\mathcal{N}=4$ to either $\mathcal{N}=2$ or $\mathcal{N}=1$.

We emphasize that using BK-type LV interactions to partially break extended SUSY results in a theory with manifest Lorentz violation. Furthermore, the details of both nonrenormalization and energy positivity are largely unchanged in the extended SUSY scenario. Thus, we can view the original $\mathcal{N}=1$ Berger-Kostelecký construction as a laboratory for exploring universal features of this class of Lorentz violating supersymmetric theories.

We speculate that Seiberg's seminal results [20] for $\mathcal{N}=2$ and $\mathcal{N}=4$ theories $^{2}$ will also continue to hold (in a sense) for BK-type theories. These theories with Lorentz violation in extended SUSY were first constructed in [6]. There are countless examples in the literature of theories that break $\mathcal{N}=4 \rightarrow \mathcal{N}=2, \mathcal{N}=4 \rightarrow \mathcal{N}=1$, or $\mathcal{N}=2 \rightarrow \mathcal{N}=1$ where the broken theory inherits many useful properties from the unbroken theory, so partial breaking BK-type LV interactions should likewise inherit many features from the unbroken theory. Our reasons are twofold: first, analyticity/holomorphy is the centerpiece of Seiberg's arguments, and we have shown that these arguments are unchanged by BKtype Lorentz violation. Second, as discussed above, uniform BK-type Lorentz violation is equivalent to a change of coordinates, and it does not seem credible that a change of coordinates, however peculiar and non-orthogonal, could introduce running couplings into a theory well known to be exactly conformal. This would be tantamount to an anomaly in the rescaling symmetry, which does not exist.

One might expect that an LV theory could develop unusual behavior rendering the powerful methods of [3] inapplicable, but such concerns prove groundless. For example, LV theories generically exhibit some form of instability at Planck-scale energies. Fortunately, these are reasonably well understood in the LV literature and can usually be dealt simply by taking the LV theory to be an effective theory with a UV-completion where Lorentz symmetry is restored at some sub-Planckian scale [5]. As long as the cutoff scale for the effective theory is sufficiently below the scale where instabilities develop, Lorentz symmetry is restored long before any instability can develop, as has been thoroughly explained in [5], for example. A second possibility is that modifying the superalgebra will render it inconsistent. For BK-type theories, this is not the case, but care must be taken lest the energy positivity theorem be destroyed.

\footnotetext{
${ }^{2}$ The so-called analytic "prepotential" of $\mathcal{N}=2$ that determines all the dynamics is only renormalized to one-loop order. The $\mathcal{N}=4$ theory is exactly conformal.
} 


\subsubsection{Extended supersymmetry and Berger-Kostelecký Lorentz violation}

We begin with a very brief review of $\mathcal{N}=2$ SUSY. For more detailed development, the reader is directed to one of the many excellent review articles available on the subject, such as $[25,26]$. This version of supersymmetry has 4 fermionic generators, $Q_{\alpha}^{a}$, where $\alpha$ is a spinor index, and $a$ simply labels the SUSY generators. After appropriate unitary transformations have been made to skew-diagonalize the central charges, the algebra of the supercharges is

$$
\begin{aligned}
\left\{Q_{\alpha}^{a}, \bar{Q}_{b \dot{\beta}}\right\} & =2\left(\sigma^{\mu}\right)_{\alpha \dot{\beta}} P_{\mu} \delta_{b}^{a} \\
\left\{Q_{\alpha}^{a}, Q_{\beta}^{b}\right\} & =2 \sqrt{2} \epsilon_{\alpha \beta} \epsilon^{a b} Z \\
\left\{\bar{Q}_{a \dot{\alpha}}, \bar{Q}_{\dot{\beta} b}\right\} & =2 \sqrt{2} \epsilon_{\dot{\alpha} \dot{\beta}} \epsilon_{a b} Z
\end{aligned}
$$

An $\mathcal{N}=2$ vector multiplet can be thought of as a standard $\mathcal{N}=1$ vector multiplet and a standard $\mathcal{N}=1$ chiral multiplet in the same representation of the gauge group. The full set of supersymmetry transformations can be deduced from the superalgebra (2.13) above, but an oversimplified heuristic is that the extra supersymmetry generators mix fields between the two $\mathcal{N}=1$ multiplets. We will use the same notation for $\mathcal{N}=2$ as we do for $\mathcal{N}=1$, with $\Phi$ denoting the $\mathcal{N}=1$ chiral superfield, $V$ denoting the $\mathcal{N}=1$ vector superfield, and components denoted $\phi(x)$ for the complex scalar field, $\psi(x)$ for the Weyl fermion, $F(x)$ for the chiral auxiliary field, $A_{\mu}$ for the real vector field, $\lambda$ for the Weyl fermion, and $D$ for the vector auxiliary field.

The $\mathcal{N}=2$ vector multiplet Lagrangian can be similarly extended from $\mathcal{N}=1$ Lagrangians. A general (not necessarily renormalizable) Lagrangian for supersymmetric gauge theories can be written as

$$
\mathcal{L}=\int d^{4} \theta K(\Phi, \bar{\Phi})+\left(\int d^{2} \theta\left(\frac{1}{4} \mathcal{W}^{\alpha} \mathcal{W}_{\alpha}+W(\Phi)\right)+\text { c.c. }\right)
$$

where $K(\Phi, \bar{\Phi})$ is a general function of the chiral superfield, $\Phi$, and its complex conjugate, $W(\Phi)$ is a holomorphic function of $\Phi$, and $\mathcal{W}_{\alpha}$ is the gauge field-strength chiral superfield, given by $\mathcal{W}_{\alpha}=-\frac{1}{4} D^{\dagger} D^{\dagger}\left(e^{-V} D_{\alpha} e^{V}\right)$. If we rescale all the fields so that the vector kinetic term is $\frac{1}{4 q^{2}} \operatorname{Tr} F_{\mu \nu} F^{\mu \nu}$, set $W(\Phi)=0$ and $K\left(\Phi, \Phi^{\dagger}\right)=\Phi^{\dagger} e^{-2 V} \Phi$, then the Lagrangian is manifestly $\mathcal{N}=2$ supersymmetric.

After eliminating the auxiliary fields in favor of their equations of motion, the $\mathcal{N}=2$ Lagrangian has the following form, expanded out in component fields [26]:

$$
\begin{aligned}
\mathcal{L}=\frac{1}{g^{2}} \operatorname{Tr}(- & \frac{1}{4} F_{\mu \nu} F^{\mu \nu}+g^{2} \frac{\theta}{32 \pi^{2}} F_{\mu \nu} \tilde{F}^{\mu \nu}+\left(D_{\mu} \phi\right)^{\dagger} D^{\mu} \phi-\frac{1}{2}\left[\phi^{\dagger}, \phi\right]^{2} \\
& \left.-i \lambda \sigma^{\mu} D_{\mu} \bar{\lambda}-i \bar{\psi} \bar{\sigma}^{\mu} D_{\mu} \psi-i \sqrt{2}[\lambda, \psi] \phi^{\dagger}-i \sqrt{2}[\bar{\lambda}, \bar{\psi}] \phi\right)
\end{aligned}
$$


where $D_{\mu}$ is the (not super) gauge covariant derivative. To add LV interactions, we follow the prescription of [1] and "twist" any derivative that acts on $\phi$ or $\psi: \partial_{\mu} \rightarrow \partial_{\mu}+k_{\mu}{ }^{\nu} \partial_{\nu}$.

$$
\begin{aligned}
\mathcal{L}=\frac{1}{g^{2}} \operatorname{Tr}(- & \frac{1}{4} F_{\mu \nu} F^{\mu \nu}+g^{2} \frac{\theta}{32 \pi^{2}} F_{\mu \nu} \tilde{F}^{\mu \nu}-i \lambda \sigma^{\mu} D_{\mu} \bar{\lambda} \\
& +\left(D_{\mu}+k_{\mu}{ }^{\nu} D_{\nu}\right) \phi^{\dagger}\left(D^{\mu}+k_{\mu}{ }^{\nu} D_{\nu}\right) \phi-\frac{1}{2}\left[\phi^{\dagger}, \phi\right]^{2}-i \bar{\psi} \bar{\sigma}^{\mu}\left(D^{\mu}+k_{\mu}{ }^{\nu} D_{\nu}\right) \psi \\
& \left.-i \sqrt{2}[\lambda, \psi] \phi^{\dagger}-i \sqrt{2}[\bar{\lambda}, \bar{\psi}] \phi\right)
\end{aligned}
$$

where we have organized the equation to emphasize the $\mathcal{N}=1$ supersymmetries. The first line of (2.16) contains all the terms for the Lagrangian of an $\mathcal{N}=1$ vector multiplet, the second an $\mathcal{N}=1$ chiral multiplet with LV interactions, and the third line contains the terms needed to combine the two multiplets into $\mathcal{N}=2$ SUSY if LV were not present. It is easy to see that this preserves gauge invariance by writing the twisted derivative as a product: $D_{\mu}+k_{\mu}{ }^{\nu} D_{\nu}=\left(\delta_{\mu}{ }^{\nu}+k_{\mu}{ }^{\nu}\right) D_{\nu}$. As in [1] this almost preserves ordinary SUSY. In the $\mathcal{N}=1[1]$ and the unbroken $\mathcal{N}=4$ theories [6], we modify the superalgebra (suppressing spinor indices):

$$
\left\{Q^{a}, \bar{Q}_{b}\right\} \rightarrow 2 \delta_{b}^{a}\left(\delta_{\mu}^{\nu}+k_{\mu}^{\nu}\right) \sigma^{\mu} P_{\nu}
$$

To implement partial SUSY breaking by LV, we promote $k_{\mu}{ }^{\nu}$ to an operator that simply multiplies fields $\phi$ and $\psi$ (the $\mathcal{N}=1$ chiral multiplet) but annihilates $A_{\mu}$ and $\lambda$ (the $\mathcal{N}=1$ vector multiplet). Invariance of the first two lines of (2.16) is obvious. Invariance of the third line is more subtle but can quickly be shown by using the fact that the LV coupling only appears in the variation of $\psi$ and is imaginary, so it will show up with opposite sign in the two terms.

An alternative way to see that (2.16) preserves $\mathcal{N}=1$ SUSY is to write the Lagrangian using superfield notation:

$$
\mathcal{L}=\frac{1}{8 \pi} \operatorname{Im} \operatorname{Tr}\left[\tau \int d^{2} \theta W^{\alpha} W_{\alpha}+2 \int d^{4} \theta \Phi^{\dagger} e^{-2 V} \Phi\right]
$$

as demonstrated in, for instance, [26]. The LV interaction is hidden within the superfields themselves using the construction of [1], so the Lagrangian appears the same as the nonLV version. However, this obscures the fact that the full $\mathcal{N}=2$ SUSY of (2.18) is broken down to $\mathcal{N}=1$. This is manifest in the on-shell component form (2.16), where the kinetic term of $\psi$ is modified by the LV interaction while that of $\lambda$ is not. A useful heuristic from [26] for identifying the extra SUSY transformations of $\mathcal{N}=2$ is to make the switch $\lambda \rightarrow \psi, \psi \rightarrow-\lambda$ in the SUSY transformation relations. It is clear from this that (2.16) does not satisfy full $\mathcal{N}=2$.

\subsubsection{A manifestly non-trivial theory with BK-type Lorentz violation}

We wish to reemphasize the most salient features of the theory described by (2.16). It is an $\mathcal{N}=1$ SUSY gauge theory with an adjoint chiral multiplet where LV interactions affect only the chiral sector. The coordinate transformation that would normally absorb the LV 
interaction into the metric in a theory with only a vector multiplet [6] or with only chiral multiplets [1] will here have the effect of moving the LV interaction from the chiral sector to the vector sector. Similar constructions are possible in $\mathcal{N}=4$ super Yang-Mills or in $\mathcal{N}=2$ theories with hypermultiplets.

Since the bulk of this paper addresses non-renormalization and energy positivity, we emphasize that the extra structure of theories with extended supersymmetry does not impair any of the $\mathcal{N}=1$ arguments. In fact, spurion analysis and constraints such as holomorphy and R-symmetry will likely introduce additional constraints on a theory using BK-type LV interactions to partially break extended SUSY. However, those constraints will depend on the particulars of the model in question. In this paper we focus only on model-independent results that apply to any model in the BK class. As such, we will work in the $\mathcal{N}=1$ theory, even though the theory is likely trivial, so as to avoid introducing any model-specific features into our results.

\section{Energy positivity in the BK construction}

Examination of the modified superalgebra relation (2.10) reveals the concern at once. The operator $\left\{Q, Q^{\dagger}\right\}$ is positive definite by construction. In traditional SUSY this guarantees energy positivity by well known arguments. With the modified superalgebra of BK-type Lorentz violation, positive definiteness of $\left\{Q, Q^{\dagger}\right\}$ can actually require negative energy if the components of $k_{\mu \nu}$ are too negative. By inspection one can see that the choice $k_{00}<-1$, for example, will require negative energy. ${ }^{3}$ Clearly the components of $k_{\mu \nu}$ must be subject to additional constraints if the positive energy theorem is to survive.

It is worth noting that ambiguities arise when defining the Hamiltonian for the Dirac equation in the presence of Lorentz violation, and that it is necessary to perform a spinor-field redefinition in order to have a hermitian Hamiltonian for Dirac particles [5]. Fortunately, the redefinition of what is meant by the "Hamiltonian" and "energy" does not impact this discussion, since the questions here relate to $p_{0}$, the space-like $p_{i}$, and the LV coupling $k_{\mu \nu}$. The phrase "energy positivity" describes the $p_{0} \geq 0$ condition, and even after redefining spinor fields, it remains true that $p_{0}$ is equal to the Hamiltonian.

In this section we take the expectation value of $\left\{Q, Q^{\dagger}\right\}$ for various generic spin-0 and spin- $1 / 2$ states and explore the constraints on $k_{\mu \nu}$ necessary to preserve the positive energy theorem.

\subsection{Constraint from spin $1 / 2$ particles at rest}

Taking the expectation value of $\left\{Q, Q^{\dagger}\right\}$ for a generic spin $1 / 2$ state, $|\psi\rangle$, yields the following modified positive energy condition:

$$
0 \leq\langle\psi|\left(\sigma^{\mu}\left(p_{\mu}+k_{\mu}^{\nu} p_{\nu}\right)|\psi\rangle\right.
$$

We are interested in constraints on $k_{\mu \nu}$ such that (3.1) guarantees $p_{0} \geq 0$, i.e. energy positivity. We will evaluate this with the assumption that $|\psi\rangle$ is a generic but normalized

\footnotetext{
${ }^{3}$ This was noted earlier in [18]. The LV coupling in that case was restricted to the special form $k_{\mu \nu}=$ $\alpha u_{\mu} u_{\nu}$, where $u_{\mu}$ was a 4 -vector of norm \pm 1 or 0 .
} 
two-component spinor, parametrized as $|\psi\rangle=\left(\begin{array}{l}a \\ b\end{array}\right)$. This yields

$$
\begin{aligned}
0 \leq & \left(p_{0}+k_{0}^{\alpha} p_{\alpha}\right)+\left(p_{3}+k_{3}^{\alpha} p_{\alpha}\right)\left(|a|^{2}-|b|^{2}\right) \\
& +2\left(p_{1}+k_{1}^{\alpha} p_{\alpha}\right) \operatorname{Re}\left(a^{*} b\right)+2\left(p_{2}+k_{2}^{\alpha} p_{\alpha}\right) \operatorname{Im}\left(a^{*} b\right) .
\end{aligned}
$$

When evaluated in the rest frame of the particle, the inequality becomes

$$
\begin{aligned}
0 \leq p_{0}( & +k_{0}^{0}+k_{3}^{0}\left(|a|^{2}-|b|^{2}\right) \\
& \left.+2 k_{1}^{0} \operatorname{Re}\left(a^{*} b\right)+2 k_{2}^{0} \operatorname{Im}\left(a^{*} b\right)\right) .
\end{aligned}
$$

This expression does not lend itself easily to analysis and completely obscures the rotational symmetry of our theory (when $k_{\mu}{ }^{\nu}$ is taken to transform appropriately). To simplify this expression, we note that the terms of (3.3) involving the $k_{i}{ }^{0}$ have the structure of a dot product of two 3 -vectors. Define $\vec{k}=\left(k_{1}{ }^{0}, k_{2}{ }^{0}, k_{3}{ }^{0}\right)$ and $\vec{a}=\left(2 \operatorname{Re}\left(a^{*} b\right), 2 \operatorname{Im}\left(a^{*} b\right),|a|^{2}-|b|^{2}\right)$. The vector $\vec{a}$ has unit norm since spinor $|\psi\rangle$ is normalized. With this replacement, equation (3.3) becomes manifestly invariant under rotations:

$$
0 \leq p_{0}\left(1+k_{0}^{0}+\vec{k} \cdot \vec{a}\right)
$$

We can now more easily explore different scenarios by considering the orientation of the vector $\vec{a}$ relative to $\vec{k}$. The case $\vec{a} \perp \vec{k}$ gives us a constraint on $k_{0}{ }^{0}$ (also mentioned above, obtained by inspection of (2.10)):

$$
k_{0}^{0}>-1
$$

where we have chosen strict inequality, since any value of $p_{0}$ would still satisfy the inequality if we chose ${k_{0}}^{0}=-1$. Once we have fixed $1+k_{0}{ }^{0}$ to be positive, the worst case scenario arises when $\vec{a}$ is chosen to be anti-parallel to $\vec{k}$. Satisfying (3.4) with positive $p_{0}$ then requires

$$
|\vec{k}|=\sqrt{\left(k_{1}{ }^{0}\right)^{2}+\left(k_{2}{ }^{0}\right)^{2}+\left({k_{3}}^{0}\right)^{2}}<1+{k_{0}}^{0} .
$$

In other words, if ${k_{0}}^{0}$ or $\vec{k}$ violate the bounds set by (3.5) and (3.6), then there exists some spinor $|\psi\rangle$ such that $p_{0}<0$ for that state in order to satisfy equation (3.1). Thus a BK theory violating either of those equations is unstable in a manner that cannot be rectified with a UV completion.

Similar constraints were explored via the dispersion relation in [18], with the restriction to the case $k_{\mu \nu}=\alpha u_{\mu} u_{\nu}$. They found that $|\alpha| \ll 1$ together with $u_{\mu} u^{\mu}= \pm 1$, 0 were sufficient to ensure consistency and that the LV terms could be treated as "small corrections". We go beyond the "small correction" case here to explore more detailed constraints for future model builders that may succeed in finding additional SUSY-scale suppression of LV coupling constants. 


\subsection{Constraints from scalar particles}

Let us now evaluate (3.1) with scalar states instead of fermions. The equation becomes

$$
\begin{aligned}
& 0 \leq\langle\phi|\{Q, \bar{Q}\}| \phi\rangle=\left\langle\phi\left|\left(2 \sum_{\mu=0}^{3}\left(p_{\mu}+k_{\mu}{ }^{\nu} p_{\nu}\right)\right)\right| \phi\right\rangle \\
&=\langle\phi| 2 {\left[p_{0}\left(1+\sum_{\mu=0}^{3} k_{\mu}{ }^{0}\right)+p_{1}\left(1+\sum_{\mu=0}^{3} k_{\mu}{ }^{1}\right)\right.} \\
&\left.+p_{2}\left(1+\sum_{\mu=0}^{3} k_{\mu}{ }^{2}\right)+p_{3}\left(1+\sum_{\mu=0}^{3} k_{\mu}{ }^{3}\right)\right]|\phi\rangle .
\end{aligned}
$$

A simple starting expression is obtained by evaluating this in the rest frame of the state $\phi$, we see that $p_{0} \geq 0$ is guaranteed only if

$$
\sum_{\mu=0}^{3} k_{\mu}^{0} \geq-1
$$

If the $\mu 0$ components of $k$ violate this inequality, then any state with scalar particles necessarily has negative energy, even when the particles are at rest.

Equation (3.7) can be used to obtain more general constraints by plugging boosted values of 4-momentum. This is discussed below in section 3.3.

As mentioned earlier, stringent phenomenological limits on the size of Lorentz violating couplings exist. For the non-supersymmetric Standard Model Extension, the results of recent literature are nicely summarized and tabulated in [8]. The supersymmetric LV parameter $k_{\mu \nu}$ is related to the non-SUSY $c$ and $k_{F}$ coefficients from [8]. The most forgiving of these constraints is $O\left(10^{-10}\right)$, so consistency constraints (3.5) and (3.6) are more or less automatically satisfied in any phenomenologically interesting theory. However, should a means be found to give Berger-Kostelecky twisted SUSY-LV couplings additional suppression of order the SUSY-breaking scale (as has been done with non-twisted SUSY-LV in [2]), such a theory would need to respect these $O(1)$ constraints.

\subsection{Constraints from moving particles}

\subsubsection{General form of constraint}

If we allow any $p_{i}$ to be non-zero, then the bound of (3.6) no longer applies. We must re-examine the constraint condition (3.2). We first consider, for simplicity, a particle moving in the 1-direction. Instead of (3.3), we now find

$$
\begin{aligned}
0 \leq & p_{0}\left(1+\left(k_{0}^{0}\right)+\left(k_{3}^{0}\right)\left(|a|^{2}-|b|^{2}\right)+2\left(k_{1}^{0}\right) \operatorname{Re}\left(a^{*} b\right)\right. \\
& \left.+2\left(k_{2}^{0}\right) \operatorname{Im}\left(a^{*} b\right)\right)+p_{1}\left(\left(k_{0}^{1}\right)+\left(k_{3}^{1}\right)\left(|a|^{2}-|b|^{2}\right)\right. \\
& \left.+2\left(1+k_{1}^{1}\right) \operatorname{Re}\left(a^{*} b\right)+2\left(k_{2}^{1}\right) \operatorname{Im}\left(a^{*} b\right)\right) .
\end{aligned}
$$


This can be simplified by use of the previously introduced vector $\vec{k}$ and a new vector that captures information about the space-space components of the second row of $\left(k_{\mu}^{\nu}\right)$. Let

$$
\vec{R}=\left(1+k_{1}{ }^{1}, k_{2}{ }^{1}, k_{3}{ }^{1}\right) .
$$

Then, mirroring the procedure that led to (3.4) we can reorganize (3.9) as

$$
0 \leq p_{0}\left(1+\left(k_{0}^{0}\right)+\vec{k} \cdot \vec{a}\right)+p_{1}\left(\left(k_{0}^{1}\right)+\vec{R} \cdot \vec{a}\right) .
$$

This makes it easy to generalize to the case of arbitrary 3-momentum by introducing one such $\vec{R}$ for each space direction. Define a more general construction of $\vec{R}^{i}$ as

$$
\left(\vec{R}^{i}\right)_{j}=\delta_{j}{ }^{i}+k_{j}{ }^{i}
$$

Then the general SUSY constraint equation for arbitrary 3-momentum is

$$
\begin{aligned}
0 \leq & p_{0}\left(1+k_{0}{ }^{0}+\vec{k} \cdot \vec{a}\right)+p_{1}\left(k_{0}{ }^{1}+\vec{R}^{1} \cdot \vec{a}\right) \\
& +p_{2}\left(k_{0}{ }^{2}+\vec{R}^{2} \cdot \vec{a}\right)+p_{3}\left(k_{0}{ }^{3}+\vec{R}^{3} \cdot \vec{a}\right)
\end{aligned}
$$

It will simplify computations to rearrange this expression into a term which is constant for all choices of spinor and a dot product term which varies from spinor to spinor as follows:

$$
0 \leq\left[p_{0}\left(1+k_{0}^{0}\right)+\sum_{i} p_{i} k_{0}^{i}\right]+\left[p_{0} \vec{k}+\sum_{i} p_{i} \vec{R}^{i}\right] \cdot \vec{a} .
$$

The worst case scenario occurs when $\left(p_{0} \vec{k}+\sum_{i} p_{i} \vec{R}^{i}\right)$ is anti-parallel to $\vec{a}$, so the strictest constraints from (3.14) are

$$
0 \leq p_{0}\left(1+k_{0}^{0}\right)+\sum_{i} p_{i} k_{0}^{i}-\left|p_{0} \vec{k}+\sum_{i} p_{i} \vec{R}^{i}\right| .
$$

Similarly, equation (3.7) now applies in full when we consider moving scalar particles.

There are two ways to further think about constraints (3.7) and (3.15): first, we can obtain the momentum by applying a particle boost (i.e. a boost that does not affect $k_{\mu \nu}$ ); second, we can impose a mass-shell condition on the momentum.

\subsubsection{Boosted particles}

Consider first a boost in the 1-direction, such that $p_{0}^{\prime}=\gamma p_{0}$ and $p_{1}^{\prime}=-v \gamma$, where $\gamma=$ $1 / \sqrt{1-v^{2}}$ as usual. Under this boost, equation (3.15) becomes

$$
\begin{aligned}
0 \leq \gamma p_{0}\left(1+k_{0}{ }^{0}\right)+-v \gamma p_{0} k_{1}{ }^{0}-\left|\gamma p_{0} \vec{k}+-v \gamma p_{0} \vec{R}^{1}\right| \\
=\gamma p_{0}\left(1+k_{0}{ }^{0}-v k_{1}^{0}-\left|\vec{k}-v \vec{R}^{1}\right|\right) \\
=\gamma p_{0}\left(1+k_{0}{ }^{0}-v k_{1}^{0}-\left[\left(k_{1}^{0}-v\left(1+k_{1}{ }^{1}\right)\right)^{2}\right.\right. \\
\left.\left.\quad+\left(k_{2}{ }^{0}-v k_{2}{ }^{1}\right)^{2}+\left(k_{3}{ }^{0}-v k_{3}{ }^{1}\right)^{2}\right]^{1 / 2}\right) .
\end{aligned}
$$


The generalization to arbitrary boosts is straightforward but unilluminating. Consistency of the twisted superalgebra then demands the components of $k_{\mu \nu}$ are chosen so that no choice of boost speed $v$ violates inequality (3.16) and its generalizations unless $v$ is high enough that the UV completion of the LV theory should be used, i.e. if $\gamma p_{0}$ is greater than the cutoff scale. We find it convenient to think about this in the following way: $k_{00}$ sets a scale for the upper limit of the absolute value of the other components of $k_{\mu \nu}$

\subsubsection{Enforcing the mass shell or dispersion relation}

Another important feature of BK-type LV theories is the modification of the dispersion relation of particles due to Lorentz violation [5] . Instead of $p^{2}=-m^{2}$, the appropriate relation is

$$
\left(\left(g^{\mu \nu}+k^{\mu \nu}\right) p_{\nu}\right)^{2}=-m^{2}
$$

as found by looking at the propagator of the fields in [5]. Lorentz violating terms in the Lagrangian change the pole of the propagator in precisely the same way as simply applying the "twisting" rule of thumb to the traditional relation. We may profitably think about this as a modification to the mass shell condition. Where we normally think of the on-shell condition as $-m^{2}=-p_{0}^{2}+\vec{p}^{2}$ (with $\vec{p}$ denoting the space-like components of momentum), the relationship is now much more complicated, with direction-dependent corrections to the old terms (arising from the diagonal elements of $k_{\mu \nu}$ ) and the addition of cross-terms (arising from off-diagonal elements of $k_{\mu \nu}$ ).

Consider, for simplicity, a particle moving purely in the 1-direction. In a Lorentz invariant theory the mass shell condition would require $p_{1}= \pm \sqrt{p_{0}^{2}-m^{2}}$. In a BK-type LV theory, that relation becomes

$$
\begin{aligned}
p_{1}= & \frac{p_{0}}{2\left(1+2 k^{11}+\left(k^{2}\right)^{11}\right)}\left(-\left(4 k^{01}+2\left(k^{2}\right)^{01}\right)\right. \\
\pm & \left(\left(4 k^{01}+2\left(k^{2}\right)^{01}\right)^{2}+4\left(1+2 k^{11}+\left(k^{2}\right)^{11}\right) \times\right. \\
& \left.\left.\left(\left(1+2 k^{00}+\left(k^{2}\right)^{00}\right)-m^{2} / p_{0}^{2}\right)\right)^{1 / 2}\right),
\end{aligned}
$$

where $\left(k^{2}\right)^{\mu \nu}=k^{\mu \alpha} k_{\alpha}{ }^{\nu}$. Note that similar but less general constraints from the dispersion relation have been obtained in [18] for the form of $k_{\mu \nu}$ considered there.

For a particle moving in a fixed direction, the mass shell condition can be used as a constraint to eliminate one of the space-like components of momentum in favor of an expression similar to (3.18).

A general boost would be parameterized by the three components of boost velocity, $v_{i}$, subject to the constraint $\sqrt{v_{1}^{2}+v_{2}^{2}+v_{3}^{2}} \leq 1$. The mass shell condition could be used to eliminate one of these degrees of freedom in favor of a constraint of the form of (3.18). The resulting expression is complicated and unilluminating. A better use of this constraint in model-building would be to first propose a choice of $k_{\mu \nu}$ then check to see whether (3.15) and (3.7) can be violated for some on-shell choice of momentum. 


\subsection{An alternate view on the positive energy constraints}

The results from section 2.2 on the non-renormalization theorem had a reassuring interpretation when viewed from the complementary perspective of the transformation that "undoes" the LV interaction in a single-sector non-extended SUSY theory: $x^{\mu \prime}=x^{\mu}-k_{\nu}^{\mu} x^{\nu}$. It would be very disturbing indeed if a simple linear coordinate transformation invalidated the non-renormalization theorem or the positive energy theorem, unless the coordinate transformation was singular or otherwise illegal. An obviously illegal choice of $k_{00}=-1$ marks a theory that transparently violates SUSY's positive energy theorem. Viewed as a coordinate transformation, it is equally obvious that the transformation is singular if any diagonal element of $k$ equals -1 . However, $k_{00}<-1$ continues to violate the positive energy theorem, whereas the coordinate transformation is no longer singular, but would change the signature of the metric. A natural first guess is that legal choices of $k_{\mu \nu}$ correspond to coordinate transformations that preserve the signature of the metric, or even the signs of all the diagonal entries. Enforcing this condition requires, for example,

$$
-2 k_{00}+k_{0}{ }^{\mu} k_{\mu 0}>1
$$

which is not obviously related to the other constraints on energy positivity from this section. We conjecture that some appropriate condition exists for $k_{\mu \nu}$ when viewed as a coordinate transformation that captures both the rest frame constraints as well as the boosted particle constraints.

\section{Non-renormalization of Nibbelink-Pospelov type LV theories}

\subsection{Review of Nibbelink-Pospelov construction}

The approach of Nibbelink and Pospelov (NP) does not alter the superalgebra. Rather, they construct LV operators that explicitly break the boost part of the superalgebra but preserve the subalgebra generated by translations and supercharges only [2]. Their construction is native to superspace and follows the usual convention of a holomorphic superpotential and non-holomorphic Kähler potential. As with the BK construction, Nibbelink and Pospelov work with 4-component Dirac spinors in the language of Wess \& Bagger [14]. We apply the same translations to the conventions of [15] as we did with the BK-construction.

Nibbelink and Pospelov classify the possible types of LV operators consistent with exact SUSY up to dimension 5. We list here for reference those LV operators relevant for SUSY gauge theories. Charged chiral superfields have only a single Kähler potential term at dimension 5 (and none at lower dimensions) [2]:

$$
N^{\mu} \bar{\Phi} e^{V} \mathcal{D}_{\mu} \Phi
$$

The gauge sector has one dimension 4 Kähler term [2]:

$$
a^{\dot{\alpha} \alpha} \operatorname{tr} \bar{W}_{\dot{\alpha}} e^{V} W_{\alpha} e^{-V}
$$

and three superpotential or gauge-kinetic terms [2]:

$$
b^{\alpha \beta} \operatorname{tr} W_{(\alpha} W_{\beta)}, \quad c^{\alpha \beta} \operatorname{tr} \Phi W_{(\alpha} W_{\beta)}, \quad T_{\mu \nu}^{\lambda} \operatorname{tr} W_{\alpha} \sigma_{\alpha \beta}^{\mu \nu} \partial_{\lambda} W_{\beta},
$$


where parentheses denote symmetrization of indices, and the gauge super field strength $W_{\alpha}$ is given by

$$
W_{\alpha}=-\frac{1}{4} D^{\dagger} D^{\dagger}\left(e^{-V} D_{\alpha} e^{V}\right)
$$

We take pains to distinguish the holomorphic superpotential $W$ from the gauge superfieldstrength $W_{\alpha}$ by always including the spinor index of the latter, even when contracted. To accommodate this convention, we have used the notation of [7] for this operator but made the spinor indices explicit.

The operators of (4.3) all represent modifications to the gauge-kinetic function. As [2] explains, only the last term of (4.3) is non-vanishing for SQED or SQCD, and even that is only true for SQED. The gauge super field strength $W_{\alpha}$ is gauge invariant only for a $\mathrm{U}(1)$ group, but replacing the ordinary spacetime derivative with a covariant derivative destroys the chirality condition, making the term not supersymmetric.

\subsection{Non-renormalization in NP-type theories}

As in the BK-construction, holomorphy is key. With the NP-construction, the superalgebra is unmodified, so holomorphy of the superpotential encodes invariance under traditional SUSY. Thus, even with NP-type LV interactions, the superpotential is immune to perturbative renormalization, and even non-perturbative renormalization is subject to tight controls. None of the well-known LV operators in the NP-construction can be added to the superpotential of SQCD, so we do not exhibit an exact superpotential calculation. An SQCD model including NP-type LV interactions in the gauge superpotential or in the Kähler potential would at most alter the running of the gauge coupling, changing Seiberg's results only by altering the coefficient of the beta function.

Weinberg [16] extends Seiberg's proof in three important ways: first, he extends the SUSY non-renormalization theorems to non-renormalizable theories, so one need not worry that higher dimension LV operators ruin these familiar results. Second, he clearly demonstrates that superpotential terms dependent on the gauge superfield strength $W_{\alpha}$ are also protected against perturbative renormalization. Third, he proves that FI terms in U(1) theories are also non-renormalized, as long as the U(1) charges of all the chiral superfields add to zero. This condition is already a well-known necessity for anomaly cancellation, and is included in the SQED model considered by [2] as well as the richer models of [7].

Weinberg's argument about the FI term has to do with gauge invariance. After promoting the FI coupling to a superfield, the FI term would not be gauge invariant if the coupling depended on any other superfield. The only gauge-invariant correction to the FI coupling arises from a diagram that vanishes when the charges are chosen as above [16].

These conditions do not change in the presence of NP-type Lorentz violation. This provides a very elegant alternative proof of the result from [7] that NP-type LV interactions do not induce a potentially divergent FI term. If the FI term is not present in the bare Lagrangian, it will not be induced in the effective Lagrangian, even by LV interactions. Conversely, if the bare Lagrangian includes an LV coupling in an FI term, that coupling will be protected against perturbative renormalization. This can be seen simply via holomorphy, without the need for computing divergent loop diagrams. 
Additionally, SUSY gauge theories are subject to powerful restrictions on the renormalization of the gauge coupling. When the fields are normalized with "holomorphic" coupling, the famous NSVZ $\beta$-function is only renormalized at one-loop order. When the fields are rescaled to canonical normalization, the $\beta$-function has a slightly different form but is exact. The original results were obtained in [21, 22]. An alternative derivation of the same results was obtained in [23]. An illuminating discussion of the alternative computation can be found in [24]. However, Weinberg offers an interesting proof of the one-loop-only renormalization result that holds for arbitrary superpotential interactions and arbitrary gauge-kinetic function couplings [16]. We briefly summarize his technique here and extend it to Lorentz-violating theories.

Weinberg begins by using Seiberg's spurion prescription, treating new coupling constants as background superfields with appropriate transformation properties for maintaining all symmetries of the lagrangian. Of particular importance will be the R-charge of the coupling and the nature of the coupling as either a chiral or vector superfield. New interactions in the Kähler potential must have vector superfield couplings, and as such these new coupling constants can only appear in non-perturbative corrections to the chiral pieces of the action, namely the superpotential and the gauge-kinetic function. So the Kähler LV interactions of (4.1) and (4.2) cannot contribute to perturbative renormalization of the effective superpotential or gauge-kinetic function. Weinberg then counts the number of graphs of different types that could contribute to a term in the effective superpotential and/or gauge-kinetic function. He considers graphs with $E_{V}$ external gaugino lines and an arbitrary number of external $\Phi$ lines, $\Phi$ being any component field of the chiral superfield(s) charged under the gauge group, and $I_{V}$ internal V-lines, $\mathrm{V}$ denoting any component of the vector superfield. Let $\mathcal{A}_{m}$ denote the number of pure gauge vertices with $m \geq 3 \mathrm{~V}$-lines, which will bring factors of the holomorphic coupling, $\tau$. Let $\mathcal{B}_{m r}$ denote the number of vertices with $m \geq r$ V-lines and any number of $\Phi$ lines which arise from extra terms in the gauge-kinetic function with $r$ factors of the vector superfield strength, $W_{\alpha}$. In the tree-level Lagrangian, the coefficient of such interactions is denoted $f_{r}$, so each of these diagrams will bring a factor of the appropriate $f_{r}$. Finally, let $\mathcal{C}_{m}$ denote the number of vertices with 2 $\Phi$ lines and $m \geq 1 \mathrm{~V}$-lines, arising from the traditional Kähler potential term, $\Phi^{\dagger} e^{-V} \Phi$. Matching gauge lines with the various types of vertices yields Weinberg's relation:

$$
2 I_{V}+E_{V}=\sum_{m \geq 3} m \mathcal{A}_{m}+\sum_{r} \sum_{m \geq r} m \mathcal{B}_{m r}+\sum_{m \geq 1} m \mathcal{C}_{m}
$$

Diagrams corresponding to the $\mathcal{A}_{m}$ and $\mathcal{C}_{m}$ terms arise from standard terms in SUSY gauge theories. Those corresponding to $\mathcal{B}_{m r}$ terms come from new interactions in the gauge kinetic function. Since $W_{\alpha}$ has R-charge +1 , the couplings $f_{r}$ must have R-charge $2-r$ for the new term to have the requisite R-charge of +2 for gauge-kinetic terms. Since the gaugino component of the vector superfield has R-charge +1 , the focus on graphs with $E_{V}$ external gauginos enforces a relationship between $E_{V}$ and the coefficients $\mathcal{B}_{m r}$ appearing in the diagram, which in turn allows one to compute the number of factors of the gauge coupling in terms of the $\mathcal{A}, \mathcal{B}, \mathcal{C}$ coefficients. Enumerating the possibilities shows that only five distinct choices of the $\mathcal{A}, \mathcal{B}, \mathcal{C}$ coefficients are legal, all of which contribute graphs independent of the gauge coupling, and all of which allow only a single non-zero 
coefficient. One of the five choices allows for a single $\mathcal{B}_{m r}=1$, which is just the tree-level contribution; the other choices only turn on an $\mathcal{A}$ or $\mathcal{C}$. Exhausting this enumeration shows that the gauge coupling only receives perturbative corrections at the one-loop level and that all coefficients $f_{r}$ in the gauge-kinetic function receive no perturbative corrections, apart from wavefunction renormalization which is not addressed by Weinberg's argument.

Thus all LV coupling constants for superpotential or gauge-kinetic function terms in the NP-construction are protected against perturbative renormalization. In [7], $\beta$-functions were computed to first-order in LV for all LV couplings relevant to $\mathcal{N}=1$ SQED. Our results are in perfect agreement with their findings for the beta function $T_{\mu \nu}^{\lambda}$ from (4.3). We take this one step further, showing that to any order in the LV couplings, this term is only subject to wavefunction renormalization.

\subsection{Berger-Kostelecký models with charged matter}

Now that we have fleshed out Weinberg's argument, we can apply it also to the BK-construction for pure gauge theories. Since the LV coupling enters into redefinitions of the vector superfield, it will be part of the gauge-kinetic function, and thus protected against perturbative corrections. It is an interesting puzzle whether the BK-construction can accommodate charged matter, as the gauge- and matter-sector LV couplings appear to have such wildly different renormalization properties. It may simply not be possible. Another, more tantalizing possibility is that quantum effects might force the LV couplings to differ in the two sectors, thereby breaking supersymmetry. A third possibility is that gauge-chiral interactions will cancel against pure chiral interactions and ultimately protect the LV coupling, $k_{\mu \nu}$, despite its presence in the Kähler potential.

\subsection{A comment on the possibility of SUSY-scale suppression of LV couplings}

There is some discrepancy in the literature over whether SUSY breaking effects can lead to additional suppression of Lorentz violating couplings. When Lorentz violation in a WessZumino model occurs via the cutoff regularization procedure as studied in [19], it is found quite generally that quantum effects rescale Lorentz violating couplings by a term proportional to $(M / \Lambda)^{2} \log (M / \Lambda)$, where $M$ is the SUSY scale and $\Lambda$ is the Lorentz-breaking scale. On the other hand, the results of [13] indicate that SUSY-scale suppression of LV couplings is incompatible with gauge theories and can only occur with neutral chiral superfields.

While each of these works looks at different models of Lorentz violation, the "no-go" results of [13] for LV SUSY gauge theories are compatible with our results. Generically, we find that LV interactions in the superpotential or the gauge-kinetic function are protected against perturbative renormalization by an extension of Seiberg's holomorphy arguments. Those concerned with fine-tuning problems will need to consider more exotic models than the original BK- and NP-constructions. The model we put forward in (2.16) is one such candidate. In that theory, the LV interactions affect only the adjoint chiral multiplet and not the gauge multiplet itself, and since the LV interaction lives in the Kähler potential for chiral superfields, it will not be protected against running. This toy model serves as "proof of concept" both that charged fields can exhibit LV interactions and that LV couplings could be brought within phenomenological limits by additional scale suppression from SUSY breaking effects. 


\section{Conclusion}

Lorentz symmetry is not a necessary ingredient in Seiberg's holomorphy arguments. Thus, Lorentz-violating SUSY theories of both Berger-Kostelecky and Nibbelink-Pospelov type preserve all the divergence-cancellation and non-renormalization aspects of traditional SUSY theories.

NP-type theories always preserve SUSY's positive energy theorem since they do not alter the superalgebra, and LV couplings in superpotential terms are protected against perturbative renormalization. While the LV couplings are still subject to non-perturbative effects, this is limited to wave-function renormalization, and Seiberg's techniques for obtaining exact quantum superpotentials continue to apply. Kähler potential LV interactions are not protected. Kähler potential as well as gauge field-strength superpotential LV interaction terms may alter the gauge-coupling beta function and in turn change some of the constants in the exponents of Seiberg's exact formulas, but in the absence of matter LV terms in the superpotential, Seiberg's exact results are altered only in a trivial way [3]. The NP construction of LV superpotential terms does not appear compatible with gauge invariance for any but abelian gauge theories, so LV terms that might have a more dramatic impact on Seiberg's results are disallowed $[2,7]$.

In BK-type theories, the single LV interaction is built into a redefinition of the superfields and the superalgebra itself. The construction is such that the LV coupling constant will only survive Grassman integration in the Kähler potential, so the superpotential remains unchanged. Seiberg's holomorphy arguments guarantee that the superpotential in BK-type theories remains non-renormalized (perturbatively), but this offers no protection to the LV coupling constant itself in Wess-Zumino models.

The positive energy theorem only continues to hold if the LV coupling, $k_{\mu \nu}$ obeys constraints (3.5), (3.6), (3.8), in addition to (3.7) and (3.15), which must hold for arbitrary on-shell momentum below the cutoff scale of the effective theory. While these constraints are many orders of magnitude less stringent than current phenomenological limits, they become important in models with $\mathrm{O}(1) \mathrm{LV}$ couplings that are suppressed as we run to lower energies. As noted above, such suppression requires different LV couplings for gauge and matter multiplets, which typically requires some level of SUSY breaking itself.

We have laid out such an example whereby BK-type LV interactions can be used to partially break extended SUSY, rendering a theory that possesses both (some) unbroken SUSY and BK-type LV interactions that are robust against coordinate transformations. We currently know of no such model that has been fully fleshed out in the literature. We save detailed investigation of such models for the future, noting for the time being that the positive energy theorem has essentially the same form regardless of the degree of supersymmetry. Our results here for the possibly trivial $\mathcal{N}=1$ BK-type models serve as a model-independent baseline set of constraints for non-trivial BK-type models using LV to partially break extended SUSY. Each specific non-trivial realization of will likely carry additional, model-specific constraints.

This work opens the door to the application of powerful modern techniques in supersymmetry, such as Seiberg's holomorphy arguments, to theories with Lorentz-violation. To our knowledge, the main body of the Lorentz violation literature has not yet employed 
these techniques. ${ }^{4}$ It would be interesting to extend the "mixed sector" BK-type approach described in (2.16) to $\mathcal{N}=4$ as well as extending NP-type theories to that degree, in order to compare with the general AdS/CFT computations of [27]. It will also be particularly interesting to consider BK-type Lorentz violation in the context of $\mathcal{N}=2$ gauge theory with matter, where the Lorentz violation affects only the matter multiplets. The machinery of Seiberg-Witten theory should apply, with SUSY breaking originating from the LV couplings rather than mass terms.

\section{Acknowledgments}

The author would like to thank A. Karch, A. Nelson, D. P. Wilson, P. McDonald, D. Colladay, M. Huber, W. Gryc, and B. Fadem for helpful conversations and comments on earlier drafts. The author gratefully acknowledges the generous support of the Office of the Provost of Muhlenberg College via a Faculty Summer Research Grant.

Open Access. This article is distributed under the terms of the Creative Commons Attribution License (CC-BY 4.0), which permits any use, distribution and reproduction in any medium, provided the original author(s) and source are credited.

\section{References}

[1] M. Berger and V.A. Kostelecky, Supersymmetry and Lorentz violation, Phys. Rev. D 65 (2002) 091701 [hep-th/0112243] [INSPIRE].

[2] S. Groot Nibbelink and M. Pospelov, Lorentz violation in supersymmetric field theories, Phys. Rev. Lett. 94 (2005) 081601 [hep-ph/0404271] [InSPIRE].

[3] K.A. Intriligator, R. Leigh and N. Seiberg, Exact superpotentials in four-dimensions, Phys. Rev. D 50 (1994) 1092 [hep-th/9403198] [INSPIRE].

[4] D. Colladay and V.A. Kostelecky, CPT violation and the standard model, Phys. Rev. D 55 (1997) 6760 [hep-ph/9703464] [INSPIRE].

[5] V.A. Kostelecky and R. Lehnert, Stability, causality and Lorentz and CPT violation, Phys. Rev. D 63 (2001) 065008 [hep-th/0012060] [INSPIRE].

[6] D. Colladay and P. McDonald, Vector Superfields and Lorentz Violation, Phys. Rev. D 83 (2011) 025021 [arXiv: 1010.1781] [INSPIRE].

[7] P.A. Bolokhov, S. Groot Nibbelink and M. Pospelov, Lorentz violating supersymmetric quantum electrodynamics, Phys. Rev. D 72 (2005) 015013 [hep-ph/0505029] [INSPIRE].

[8] V.A. Kostelecky and N. Russell, Data tables for Lorentz and CPT Violation, Rev. Mod. Phys. 83 (2011) 11 [arXiv:0801.0287] [InSPIRE].

[9] N. Seiberg, The power of holomorphy: Exact results in 4-D SUSY field theories, hep-th/9408013 [inSPIRE].

\footnotetext{
${ }^{4}$ Two recent works $[27,28]$ approach Lorentz violation in a context native to the AdS/CFT correspondence and quite different at first appearance from the well-trodden paths initially blazed by Kostelecky and collaborators. While SUSY is an important ingredient in any AdS/CFT construction, the absence of superfields in $\mathcal{N}=4$ SUSY necessarily renders these approaches quite different from both the BK- and the NP-constructions.
} 
[10] D. Colladay and P. McDonald, One-Loop Renormalization of the Electroweak Sector with Lorentz Violation, Phys. Rev. D 79 (2009) 125019 [arXiv:0904.1219] [INSPIRE].

[11] D. Colladay and P. McDonald, One-Loop Renormalization of QCD with Lorentz Violation, Phys. Rev. D 77 (2008) 085006 [arXiv: 0712.2055] [INSPIRE].

[12] D. Colladay and P. McDonald, One-Loop Renormalization of Pure Yang-Mills with Lorentz Violation, Phys. Rev. D 75 (2007) 105002 [hep-ph/0609084] [INSPIRE].

[13] D. Redigolo, On Lorentz-Violating Supersymmetric Quantum Field Theories, Phys. Rev. D 85 (2012) 085009 [arXiv:1106. 2035] [INSPIRE].

[14] J. Wess and J. Bagger, Supersymmetry and supergravity, Princeton University Press, Princeton U.S.A. (1992), pg. 259.

[15] S.P. Martin, A supersymmetry primer, hep-ph/9709356 [INSPIRE].

[16] S. Weinberg, Nonrenormalization theorems in nonrenormalizable theories, Phys. Rev. Lett. 80 (1998) 3702 [hep-th/9803099] [INSPIRE].

[17] A. Ferrero and B. Altschul, Renormalization of Scalar and Yukawa Field Theories with Lorentz Violation, Phys. Rev. D 84 (2011) 065030 [arXiv:1104.4778] [INSPIRE].

[18] C. Farias, A. Lehum, J. Nascimento and A.Y. Petrov, On the superfield supersymmetric aether-like Lorentz-breaking models, Phys. Rev. D 86 (2012) 065035 [arXiv:1206.4508] [INSPIRE].

[19] P. Jain and J.P. Ralston, Supersymmetry and the Lorentz fine tuning problem, Phys. Lett. B 621 (2005) 213 [hep-ph/0502106] [INSPIRE].

[20] N. Seiberg, Supersymmetry and Nonperturbative $\beta$-functions, Phys. Lett. B 206 (1988) 75 [INSPIRE].

[21] M.A. Shifman and A. Vainshtein, Solution of the Anomaly Puzzle in SUSY Gauge Theories and the Wilson Operator Expansion, Nucl. Phys. B 277 (1986) 456 [INSPIRE].

[22] V. Novikov, M.A. Shifman, A. Vainshtein and V.I. Zakharov, Exact Gell-Mann-Low Function of Supersymmetric Yang-Mills Theories from Instanton Calculus, Nucl. Phys. B 229 (1983) 381 [INSPIRE].

[23] N. Arkani-Hamed and H. Murayama, Holomorphy, rescaling anomalies and exact $\beta$-functions in supersymmetric gauge theories, JHEP 06 (2000) 030 [hep-th/9707133] [INSPIRE].

[24] M.J. Strassler, An unorthodox introduction to supersymmetric gauge theory, hep-th/0309149 [INSPIRE].

[25] A. Bilal, Duality in $N=2$ SUSY SU(2) Yang-Mills theory: A pedagogical introduction to the work of Seiberg and Witten, hep-th/9601007 [INSPIRE].

[26] L. Álvarez-Gaumé and S. Hassan, Introduction to $S$ duality in $N=2$ supersymmetric gauge theories: A pedagogical review of the work of Seiberg and Witten, Fortsch. Phys. 45 (1997) 159 [hep-th/9701069] [INSPIRE].

[27] E. Kiritsis, Lorentz violation, Gravity, Dissipation and Holography, JHEP 01 (2013) 030 [arXiv:1207.2325] [INSPIRE].

[28] R. Sundrum, Gravitational Lorentz Violation and Superluminality via AdS/CFT Duality, Phys. Rev. D 77 (2008) 086002 [arXiv:0708.1871] [INSPIRE]. 